\title{
Efeito do Co-Intercalante C/S-13-Docosenamida na Morfologia e Propriedades Mecânicas de Compósitos Polipropileno/Argila Organofílica
}

\author{
Tatianny Soares Alves, Renata Barbosa \\ Universidade Federal do Piauí, UFPI \\ Clarissa Coussirat Angrizani, Maria Goretti Coutinho, Laura Hecker de Carvalho
Universidade Federal de Campina Grande, UFCG
}

Eduardo Luis Canedo

PolyTech, Prospect CT, USA

\begin{abstract}
Resumo: Compósitos de PP/argila/PP-g-MA/erucamida contendo 5\% (em peso) de argila montmorilonita organofílica (Cloisite 20A), 5 e 15\% de PP-g-MA como agente compatibilizante e 0, 0,5\% e 1\% de cis-13-docosenamida (erucamida) como agente co-intercalante, foram preparados por intercalação no estado fundido. Concentrados de argila e compatibilizantes foram processados em misturador interno e em seguida diluídos com a matriz de polipropileno em extrusora de dupla rosca corrotacional. Os compósitos foram caracterizados através das propriedades mecânicas, difração de raios X e microscopia óptica. Os resultados indicaram o efeito reforçante da argila organofilizada. A presença de co-intercalante aumentou significativamente a distância interlamelar da argila em todos os compósitos, em geral, não afetando notavelmente o comportamento mecânico dos compósitos. Por microscopia óptica foi possível observar o efeito dispersivo do perfil de rosca utilizado, que promoveu a quebra dos aglomerados de argila.
\end{abstract}

Palavras-chave: Polipropileno, nanocompósitos, co-intercalante, propriedades mecânicas.

\section{Effect of CIS-13-Docosenamide Cointercalation on the Morphology and Mechanical Properties of Polypropylene/Organoclay Composites}

\begin{abstract}
PP/organoclay/PP-g-MA/erucamide composites with 5\% commercial organo-montmorillonite (Cloisite 20A), 5 and $15 \%$ maleated polypropylene as compatibilizer, and $0,0.5$ and $1 \%$ erucamide as a co-intercalating agent were prepared by melt intercalation. A masterbatch of compatibilizer and organoclay was compounded in an internal mixer and then diluted in the polypropylene matrix in a corotating twin-screw extruder. The compounds were characterized by x-ray diffraction, optical microscopy and mechanical properties. Results indicate that the organoclay acted as a reinforcer. Addition of the co-intercalating agent significantly increased the interlayer spacing of the clay, without notably affecting the mechanical behavior of the composites. Optical microscopy showed the dispersive effect of the screw profile used, which promoted the breaking of clay agglomerates.
\end{abstract}

Keywords: Polypropylene, nanocomposites, cointercalant, mechanical properties.

\section{Introdução}

Nanocompósitos são definidos como materiais compósitos onde a fase reforçante dispersa tem pelo menos uma das suas dimensões, da ordem de $1 \mathrm{~nm}$ $\left(10^{-9} \mathrm{~m}\right)$. Os nanocompósitos apresentam, em baixos teores de carga (1-5\%), propriedades equivalentes ou até mesmo superiores às exibidas por compósitos convencionais preparados com teores de carga bem mais elevados (20-50\%). A preparação de nanocompósitos poliméricos de matrizes apolares tem sido descrita em três estágios sucessivos ${ }^{[1]}$ que incluem: a modificação da carga; a preparação de um concentrado e a diluição do concentrado. Para o caso particular de nanocompósitos de PP/MMT, onde a MMT é modificada organicamente com um sal de amônio quaternário e o PP é compatibilizado com PP-g-MA os estágios de preparação são:
- Primeiro estágio: organofilização da montmorilonita com um sal de amônio quaternário para obter OMMT;

- Segundo estágio: preparação de um concentrado da OMMT com agente compatibilizante PP-g-MA para obter um nanocompósito intercalado;

- Terceiro estágio: diluição do concentrado com a matriz de PP para obter (eventualmente) um nanocompósito esfoliado.

O esquema de preparação dos nanocompósitos é ilustrado na Figura 1.

Para o caso de uma organomontmorilonita (OMMT) comercial, o esquema é reduzido a dois estágios (intercalação do PP-g-MA, esfoliação do concentrado na matriz de PP), que muitas vezes são realizados de

Autor para correspondência: Tatianny Soares Alves, Programa de Pós-Graduação em Ciência dos Materiais, Centro de Ciências da Natureza, Universidade Federal do Piauí - UFPI, Campus Universitário Ministro Petrônio Portella, Av. Ininga, s/n, CEP 64049-550, Teresina, PI, Brasil, e-mail: tsaeng3@yahoo.com.br 
forma conjunta, em um estágio único de processamento ${ }^{[2]}$ ou alimentando os insumos sucessivamente no mesmo equipamento ${ }^{[3]}$.

Ainda que o esquema anterior seja frequentemente utilizado como modelo, na prática o produto final é raramente um nanocompósito completamente esfoliado, mas uma mistura de compósitos intercalados e esfoliados. A literatura sobre o processamento de nanocompósitos PP/MMT nas últimas duas décadas, relata os esforços dos pesquisadores em obter um maior grau de esfoliação, manipulando a formulação (diferentes tipos e teores de OMMT, diferente tipo e teor de agente compatibilizante) e as condições de processamento. Ainda com o objetivo de melhorar a interação entre polímero e argila, estudos recentes têm avaliado a co-intercalação de moléculas orgânicas polares (por exemplo: amidas) na formação de nanocompósitos de polipropileno ${ }^{[4,5]}$.

A dispersão da argila na matriz polimérica e as propriedades dos nanocompósitos, obtidos por mistura no estado fundido, dependem de interações específicas polímero-argila, que podem ser incrementadas através da adição de agentes compatiblizantes e co-intercalantes, mas também são fortemente influenciadas pelo equipamento utilizado e pelas condições de processamento, tais como perfil e velocidade da rosca, temperatura do fundido, bem como pelas características do polímero como sua viscosidade, massa molecular e distribuição de massa molar. Estas variáveis afetam tanto a cinética de intercalação das cadeias de polipropileno para o interior das lamelas da argila quanto a viscosidade do fundido, alterando o nível de deformação e as tensões sofridas pelo polímero durante o processamento ${ }^{[6,7,8,9]}$. Os nanocompósitos podem ser preparados, via técnica de intercalação no estado fundido, através de uma variedade de dispositivos que geram deformações e tensões de cisalhamento. Entre os equipamentos utilizados preferencialmente, os misturadores internos com rotores contrarrotacionais e as extrusoras modulares de dupla rosca corrotacionais completamente entrelaçadas, têm mostrado uma maior flexibilidade e eficiência na dispersão da $\operatorname{argila}{ }^{[10]}$.

O presente trabalho reporta os efeitos da incorporação de um agente compatiblizante e de um agente co-intercalante na obtenção de nanocompósitos PP/argila organofílica.

\section{Experimental}

\section{Materiais}

Os compósitos investigados foram do tipo PP/PP-gMA/argila organofílica/agente co-intercalante.

Como matriz polimérica foi utilizado o homopolímero PPH301 (IF = 10). O índice de fluidez citado corresponde ao fluxo de fundido (MFR) em dg/min medido a $230{ }^{\circ} \mathrm{C}$ com um peso de $2,16 \mathrm{~kg}$ em plastômetro padronizado de acordo com a norma ASTM D1238.

O agente compatibilizante, um polipropileno funcionalizado (copolímero enxertado de propileno e anidrido maleico) fornecido pela Chemtura, sob o nome comercial de Polybond 3200, com 1,0\% de anidrido maleico (nominal) e índice de fluidez de $115 \mathrm{dg} / \mathrm{min}$ (ASTM D1238, $190{ }^{\circ} \mathrm{C} / 2,16 \mathrm{Kg}$ ), foi utilizado como agente compatibilizante padrão em teores de 5 e $15 \%$. A carga empregada foi a montmorilonita organicamente modificada, Cloisite 20A, fornecida pela Southern Clay Products. O co-intercalante usado foi a cis-13-docosenamida $\left(\mathrm{C}_{22} \mathrm{H}_{43} \mathrm{NO}\right.$, massa molar $\mathrm{M}=337,6 \mathrm{~g}$ ), em teores de 0,5 e $1,0 \%$, de nome comercial Armoslip E Powder da AkzoNobel, fornecido pela Convip.

\section{Métodos}

Concentrados foram preparados em misturador interno, Rheomix 3000, com câmara apresentando volume livre $310 \mathrm{~cm}^{3}$ e rotores do tipo "roller". Um fator de preenchimento de $70 \%$ foi utilizado em todos os casos e o misturador foi operado a $190{ }^{\circ} \mathrm{C}$ e $60 \mathrm{rpm}$ durante 7 minutos. Em seguida, os concentrados foram diluídos em extrusora dupla rosca corrotacional modular, marca NZ, modelo SJ-20, com diâmetro de 22 mm, L/D = 38, e fator de forma 1,48 , sob velocidade de $240 \mathrm{rpm}$, com vazão de alimentação de $11 \mathrm{~kg} / \mathrm{h}$. Para melhor avaliar o efeito das composições individualmente, foram processadas blendas de PP-g-MA/ PP e matriz pura sob as mesmas condições de extrusão que os demais compósitos.

Os compósitos obtidos foram moldados em injetora FLUIDMEC na forma de corpos de prova para ensaios de tração e impacto, segundo as dimensões preconizadas pelas normas ASTM D 638 e ASTM D 256, respectivamente. A temperatura de injeção foi de

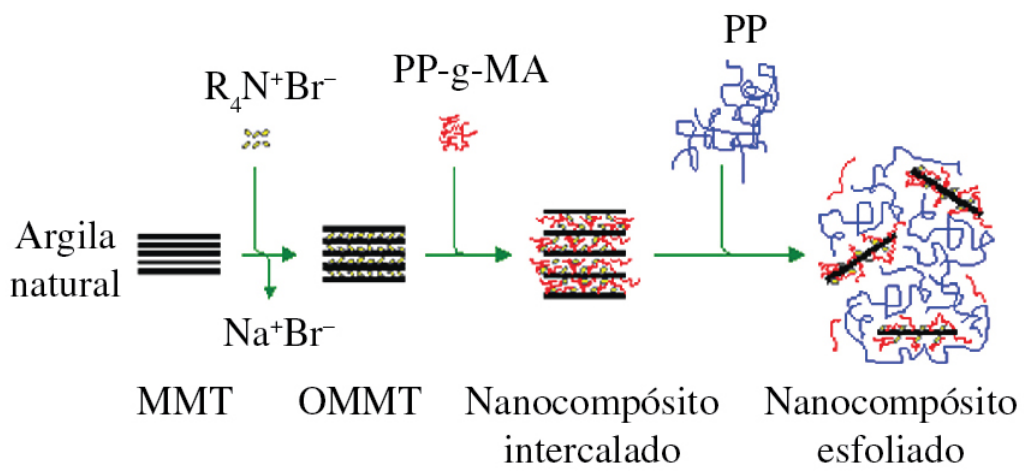

Figura 1. Estágios da preparação de um nanocompósito PP/MMT por intercalação no estado fundido, de acordo com esquema (hipotético) clássico. Adaptado de Kawasumi et al. ${ }^{[1]}$. 
$200{ }^{\circ} \mathrm{C}$, em todas as zonas, com tempo de resfriamento de 25 segundos e temperatura de molde fechado de $20^{\circ} \mathrm{C}$.

Todas as composições dos masterbatches e as respectivas diluições em extrusora são indicadas na Tabela 1, onde a partir dos quatro concentrados foram obtidos quatro composições distintas.

\section{Caracterização}

\section{Difração de raios $X(D R X)$}

A distância interplanar basal da argila e dos compósitos foi avaliada através da difração de raios-X utilizando equipamento da marca Shimadzu. A fonte da radiação incidente foi $\mathrm{Cu}_{\mathrm{K} \alpha}$ com comprimento de onda de $0,154 \mathrm{~nm}$. Os dados foram coletados em uma velocidade de varredura de $2 \%$ min e faixas angulares $(2 \theta)$ entre $1,5^{\circ}$ e $10^{\circ}$

\section{Microscopia óptica (MO)}

A técnica de microscopia óptica foi utilizada como método preliminar de observação do grau de dispersão da carga nas matrizes de polipropileno e do efeito do tipo de protocolo utilizado para processamento. Foram utilizados corpos de prova injetados para a realização das análises. Foi utilizado um microscópio trinocular da marca Lederer \& Avancini, operando no modo de transmissão.

\section{Propriedades mecânicas}

Os ensaios de tração e impacto foram realizados segundo a norma ASTM D 638, em uma máquina de ensaios mecânicos de marca Lloyd, modelo LR 10K. Os testes foram conduzidos à temperatura ambiente. Os testes de impacto foram realizados conforme a norma ASTM D 256 em um aparelho de impacto da marca Ceast modelo Resil 5.5, operando com martelo de 2,75 J. Os resultados médios de 10 corpos de prova foram reportados.

\section{Resultados e Discussão}

\section{Difração de raios $X$}

A Figura 2 ilustra os difratogramas obtidos para os compósitos processados em extrusora com distintos percentuais de compatibilizante e co-intercalante. $\mathrm{Na}$ Tabela 2 os valores numéricos das reflexões basais e da distância interplanar $d_{(001)}$ são apresentados.

De maneira geral, observa-se que a utilização de agente compatibilizante PP-g-MA aumentou a distância interplanar basal de todos os compósitos, indicando a formação de estruturas intercaladas. Os resultados sugerem ainda, que o PP- $g$-MA facilitou a delaminação e a dispersão da argila na matriz de polipropileno, mas que em teores elevados o compatibilizante teria produzido um efeito lubrificante durante o processamento dos compósitos, não favorecendo uma esfoliação da argila. Resultados semelhantes foram observados por Morales et al. ${ }^{[11]}$ e Lee et al. ${ }^{[12]}$ ao avaliarem compósitos formados a base de argila organofílica, polipropileno e agente compatibilizante.

A presença de outro aditivo polar (erucamida), levou ao deslocamento dos picos de difração (001) para ângulos mais baixos, passando de $3,62^{\circ}$, referente a argila pura, para $2,14^{\circ}$ referente ao sistema a base de $0,5 \%$ de erucamida, acarretando em um considerável aumento da distância interplanar basal, que passou de 2,43 para 4,12 nm. Esse comportamento pode ser atribuído à intercalação das moléculas polares da erucamida, através da formação de pontes de hidrogênio entre o grupo amida $\left(\mathrm{CONH}_{2}\right)$ presente no aditivo, com os íons das camadas da argila. Em todos os compósitos contendo erucamida em sua composição foram observados os maiores percentuais de aumento na distância basal. Também foi possível observar o aumento na intensidade do pico dos compósitos com erucamida, em especial para aqueles com maior percentual deste aditivo. Aumentos na distância interplanar basal e o aparecimento de um

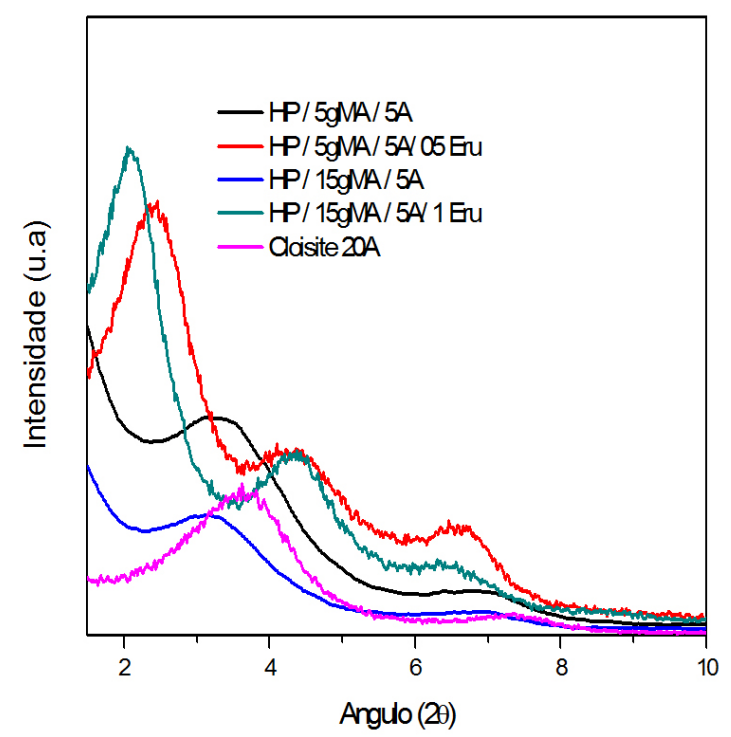

Figura 2. Difratograma de raios $\mathrm{X}$ dos compósitos a base de homopolímero.

Tabela 1. Composição dos concentrados e compósitos (\% em massa).

\begin{tabular}{|c|c|c|c|c|c|c|c|}
\hline \multirow[t]{2}{*}{ Código } & \multicolumn{3}{|c|}{ Concentrado (\%) } & \multicolumn{4}{|c|}{ Compósito (\%) } \\
\hline & gMA & $\mathbf{A}$ & Eru & HP & gMA & $\mathbf{A}$ & Eru \\
\hline $\mathrm{K} 1$ & 50,0 & 50,0 & 0 & 90,0 & 5,0 & 5,0 & 0 \\
\hline $\mathrm{K} 2$ & 75,0 & 25,0 & 0 & 80,0 & 15,0 & 5,0 & 0 \\
\hline $\mathrm{K} 3$ & 47,6 & 47,6 & 4,8 & 89,5 & 5,0 & 5,0 & 0,5 \\
\hline $\mathrm{K} 4$ & 71,4 & 23,8 & 4,8 & 74,0 & 15,0 & 5,0 & 1,0 \\
\hline
\end{tabular}

K: concentrado - HP: polipropileno PPH 301 - gMA: compatibilizante Polybond 3200 - A: Cloisite 20A - Eru: erucamida. 
segundo pico e um ombro entre 6 e $7^{\circ}$ foram obtidos por Ratnayake et al. ${ }^{[4]}$ para compósitos a base de polipropileno homopolímero, argila organofílica, compatibilizante PP-g-MA (Polybond 3200) e erucamida. A presença de picos ou ombros em ângulos $2 \theta$ entre $3^{\circ}$ e $8^{\circ}$ são interpretados como reflexões de segunda e terceira ordem no plano (001). Ainda segundo os autores, em baixos percentuais, as moléculas de erucamida ficam presas no interior da argila, evitando a migração para a superfície.

A presença da erucamida aumentou significativamente os níveis de intercalação em comparação aos compósitos preparados pelos métodos convencionais contendo apenas PP- $g$-MA.

\section{Propriedades mecânicas}

A Tabela 3 apresenta o comportamento mecânico em tração dos compósitos preparados em extrusora dupla rosca corrotacional.

As blendas de PP- $g$-MA/PP apresentaram aumentos no módulo de elasticidade; a resistência a tração não foi alterada com o aumento do teor de compatibilizante e a resistência ao impacto foi reduzida para ambos os teores de compatibilizante investigados, sendo a maior redução observada para os sistemas com $15 \%$ de PP-g-MA. A incorporação de argila e do compatibilizante provocou aumentos do módulo de elasticidade, com ganhos de até $40 \%$, obtidos com a incorporação de PP-g-MA e argila organofílica na proporção 1:1. O aumento do teor de PP- $g$-MA nos sistemas, considerando a incerteza nas medições, não promoveu alterações significativas no módulo de elasticidade dos compósitos estudados, incrementando em apenas $10 \%$ o ganho nesta propriedade quando comparado ao sistema com 5\% de agente compatibilizante. Acredita-se que a presença do agente compatibilizante tenha facilitado a ação reforçante da argila por meio de uma maior intercalação na argila como confirmado por difração de raios - x. Resultados semelhantes foram obtidos por Paiva \& Morales $^{[13]}$. A adição de erucamida elevou discretamente o módulo

Tabela 2. Picos de reflexão e distância interplanar basal $\mathrm{d}_{(001)}$ dos compósitos.

\begin{tabular}{lcccc}
\hline \multirow{2}{*}{ Compósitos } & \multicolumn{5}{c}{$\mathbf{2 \theta}\left({ }^{\circ}\right)$} & $d_{(001)}$ \\
\cline { 2 - 5 } & $\mathbf{1}$ & $\mathbf{2}$ & $\mathbf{3}$ & $\mathbf{( n m )}$ \\
\hline $\mathrm{C} 20 \mathrm{~A}$ & 3,62 & 7,34 & --- & 2,43 \\
$\mathrm{HP} / 5 \mathrm{gMA} / 5 \mathrm{~A}$ & 3,07 & 6,82 & & 2,87 \\
$\mathrm{HP} / 5 \mathrm{gMA} / 5 \mathrm{~A} / 05 \mathrm{Eru}$ & 2,14 & 3,91 & 6,34 & 4,12 \\
$\mathrm{HP} / 15 \mathrm{gMA} / 5 \mathrm{~A}$ & 3,15 & 7,04 & --- & 2,80 \\
$\mathrm{HP} / 15 \mathrm{gMA} / 5 \mathrm{~A} / 1$ Eru & 2,24 & 4,56 & 6,62 & 3,94 \\
\hline
\end{tabular}

de elasticidade em relação à matriz polimérica pura e as blendas com PP- $g$-MA, mas quando comparados os compósitos contendo apenas PP- $g$-MA e argila, o co-intercalante promoveu ganhos de propriedade inferiores.

A resistência à tração também foi afetada pela presença de argila e dos agentes compatibilizante e co-intercalante, mas em menor intensidade. Aumentos de $11 \%$ foram obtidos para os compósitos contendo maior percentual de compatibilizante sem a presença da erucamida, quando comparado à matriz polimérica pura e as blendas com PP- $g$-MA. Para as demais composições, não foram observadas variações relevantes de propriedade.

A resistência ao impacto foi a propriedade mais afetada com o aumento do percentual de compatibilizante, sofrendo reduções superiores a $50 \%$, na presença de argila e com maior percentual de compatibilizante. Para as blendas, a maior redução foi observadas quando o maior percentual de PP- $g$-MA foi utilizado. Dois fatores podem ter contribuído para a redução da tenacidade dos compósitos, na medida em que o teor de PP- $g$-MA aumentou: baixo peso molecular do compatibilizante e redução da formação da fase $\beta$, desenvolvida em condições favoráveis de processamento e que apresenta forte relação com a resistência ao impacto. Por outro lado, o aumento do percentual de erucamida, promoveu o aumento da resistência ao impacto, comparativamente ao compósito contendo apenas compatibilizante e argila e às blendas. Esse comportamento pode ser atribuído a uma melhor dispersão da carga na matriz, facilitando a transferência de tensões, com melhor absorção da energia de impacto e redução na propagação de microfissuras.

Acredita-se que uma redução na viscosidade dos sistemas, causada pela adição de maior quantidade de compatibilizante de elevado índice de fluidez se comparado à matriz $(115 \mathrm{~g} / 10 \mathrm{~min}$ vs $10 \mathrm{~g} / 10 \mathrm{~min})$, não tenha favorecido a quebra dos aglomerados de partículas de argila, não promovendo a dispersão e a ação reforçante da carga. Este resultado indica que a resistência ao impacto é fortemente dependente do teor de compatibilizante utilizado e, possivelmente, do seu índice de fluidez em relação à matriz. Resultados semelhantes foram obtidos por García-López et al. ${ }^{[14]}$, que observou melhores resultados para teores de compatibilizante entre 5 e $10 \%$. Eteläaho et al. ${ }^{[15]}$ avaliaram os efeitos da argila organofilizada (sem o uso de compatibilizantes) nas propriedades mecânicas dos compósitos. Os autores reportaram aumentos de $12 \%$ e $13 \%$ no módulo de elasticidade e resistência ao impacto, respectivamente.

Tabela 3. Propriedades mecânicas dos compósitos.

\begin{tabular}{lccc}
\hline \multicolumn{1}{c}{ Compósitos } & Módulo de elasticidade (MPa) & Resistência a tração (MPa) & Resistência ao impacto $(\mathbf{J} / \mathbf{m})$ \\
\hline HP puro & $1182,6 \pm 31,0$ & $27,4 \pm 0,3$ & $23,4 \pm 1,4$ \\
HP/5gMA & $1319,4 \pm 36,0$ & $30,1 \pm 0,9$ & $20,2 \pm 1,5$ \\
HP/5gMA/5A & $1664,8 \pm 63,8$ & $29,8 \pm 0,8$ & $24,1 \pm 1,7$ \\
HP/5gMA/5A/05 Eru & $1569,2 \pm 104,5$ & $31,0 \pm 0,5$ & $27,3 \pm 1,9$ \\
HP/15gMA & $1263,3 \pm 36,4$ & $28,4 \pm 0,8$ & $17,7 \pm 1,7$ \\
HP/15gMA/5A & $1700,2 \pm 100,2$ & $31,7 \pm 1,1$ & $10,5 \pm 0,9$ \\
HP/15gMA/5A/1 Eru & $1333,3 \pm 72,2$ & $27,6 \pm 0,9$ & $26,0 \pm 1,5$ \\
\hline
\end{tabular}




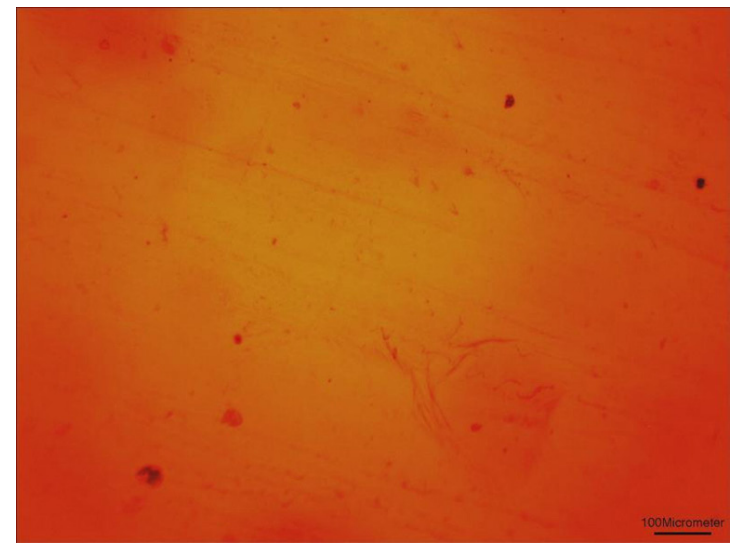

(a)

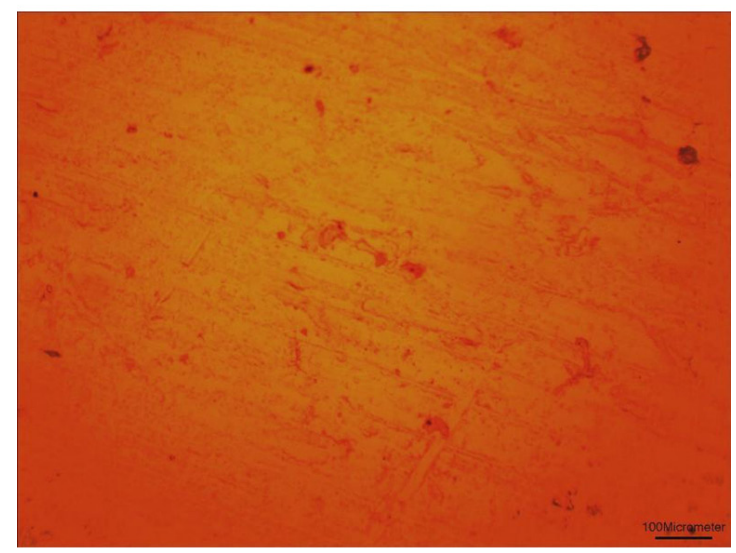

(b)

Figura 3. Micrografias ampliadas $40 \mathrm{X}$ do masterbatch: (a) destinado a composição final contendo 5\%PP-gMA/5\%argila/0,5\% Erucamida e (b) destinado a composição final contendo 15\%PP-g-MA/5\%argila/1,0\% Erucamida.

\section{Microscopia óptica}

As Figuras 3 e 4 ilustram o efeito da composição na dispersão da argila nas matrizes de polipropileno homopolímero.

Através das micrografias foi possível observar a presença de aglomerados de argila em todas as composições e sob diferentes etapas do processamento, semelhante ao observado por Rodrigues ${ }^{[16]}$. Carastan ${ }^{[17]}$, para sistemas de PS/Cloisite $15 \mathrm{~A}$, e Mohan et al. ${ }^{[18]}$ e Xie et al. ${ }^{[19]}$ para sistemas PP/Cloisite $15 \mathrm{~A}$ e PP/Cloisite 20A, respectivamente, também relataram a presença de aglomerados de argila com dimensões variadas. Os compósitos após extrusão apresentaram redução do tamanho dos aglomerados, com reflexo nas propriedades mecânicas avaliadas, e uma pequena mudança no formato dos aglomerados foi observada ao ser incorporada a matriz polimérica no momento da extrusão, tendo alguns deles formatos mais alongados. $\mathrm{O}$ aumento do percentual de erucamida tendeu a reduzir os tamanhos dos aglomerados, sugerindo uma maior facilidade de intercalação deste aditivo nas lamelas da argila.

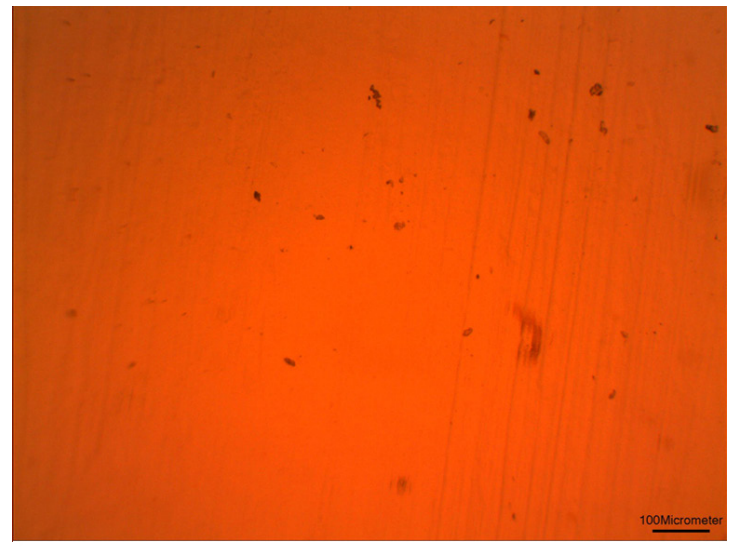

(a)

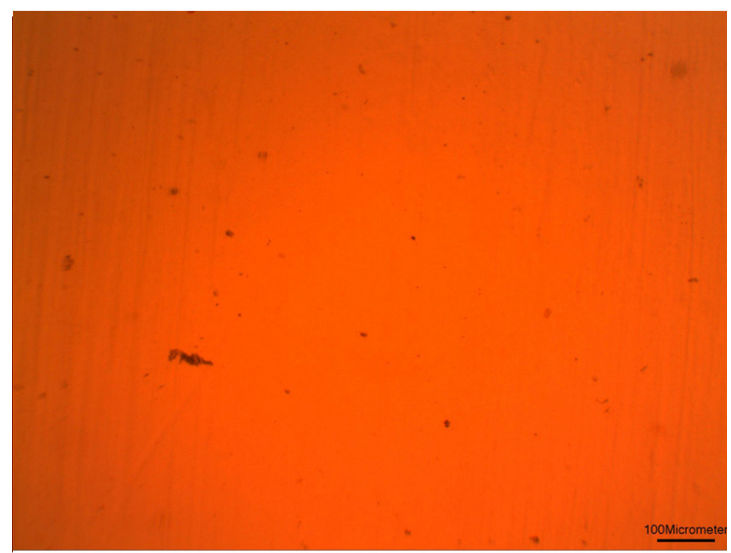

(b)

Figura 4. Micrografias ampliadas 40X: (a) HP/5gMA/5A/05 Eru e (b) HP/ 15gMA/5A/1 Eru (ambos processados via extrusão).

\section{Conclusão}

A presença de um co-intercalante polar favoreceu a dispersão da argila organofílica, promovendo um efeito mais pronunciado nos níveis de intercalação dos compósitos, com aumentos significativos na distância basal, refletindo nas propriedades mecânicas.

As propriedades mecânicas (módulo de elasticidade, resistência a tração) mostram aumentos com a incorporação de argila associada ao compatibilizante, que sendo ao ser adicionado apenas ao polipropileno não favoreceu ganhos significativos de propriedades. Dentre as propriedades mecânicas avaliadas, a resistência ao impacto foi a propriedade mecânica mais afetada pelas variações de composição: reduziu com a presença do compatibilizante, especialmente quando em maior percentual e com argila; aumentou com a presença da erucamida em ambas as proporções. A erucamida suavizou o efeito concentrador de tensão da carga frente à solicitação por impacto. 


\section{Agradecimentos}

Os autores agradecem a Braskem pela doação da matriz polimérica e a Convip pelo fornecimento da erucamida. Ao Laboratório de Caracterização de Materiais da UFCG e ao Laboratório Interdisciplinar de Materiais Avançados da UFPI. Ao CNPq pela bolsa de produtividade em pesquisa de Carvalho e pelo apoio financeiro aos projetos de número 477808/2009-3 e 481093/2011-7.

\section{Referências Bibliográficas}

1. Kawasumi, M.; Hasegawa, N.; Kato, M.; Usuki, A. \& Okada, A. - Macromolecules, 30, p.6333 (1997). http:// dx.doi.org/10.1021/ma961786h

2. Dennis, H. R.; Hunter, D. L.; Chang, D.; Kim, S.; White, J. L.; Cho, J. W. \& Paul, D. R. - Polymer, 42, p.9513 (2001). http://dx.doi.org/10.1016/S0032-3861(01)00473-6

3. Zhu, L. \& Xanthos, M. - J. Appl. Polym. Sci., 93, p.1891 (2004). http://dx.doi.org/10.1002/app.20658

4. Ratnayake, U. N.; Haworth, B. \& Hourston, J. - J. Appl. Polym. Sci., 112, p.320 (2009). http://dx.doi.org/10.1002/ app. 29371

5. Haworth, B. \& Ratnayake, U. N. - Intern. Polym. Proc, 26, p.91 (2011). http://dx.doi.org/10.3139/217.2409

6. Rauwendaal, C. - "Polymer Extrusion", 4th ed, Hanser, Munich (2001).

7. Tadmor Z. \& Gogos, C. G. - "Principles of Polymer Processing", 2nd ed, Wiley-Interscience, New York (2006).

8. Osswald, T. \& Hernández-Ortiz, J. P. - "Polymer Processing - Modeling and Simulation", Hanser, Munich (2006). http://dx.doi.org/10.3139/9783446412866

9. Manas-Zloczower, I. (ed.) - "Mixing and Compounding of Polymers - Theory and Practice", 2nd ed, Hanser, Munich (2009). http://dx.doi.org/10.3139/9783446433717
10. Alves, T. S. - "Nanocompósitos PP/bentonita: efeito das condições de processamento e viscosidade da matriz", Dissertação de Mestrado, Universidade Federal de Campina Grande, Brasil (2010).

11. Morales, A. R.; Paiva, L. B.; Zattarelli, D. \& Guimarães, T. R. - Polímeros, 22, p.54 (2012). http://dx.doi.org/10.1590/ S0104-14282012005000004

12. Lee, E. C.; Mielewski, D. F. \& Baird, R. J. - Polym. Eng. Sci., 44, p.1773 (2004). http://dx.doi.org/10.1002/ pen. 20179

13. Paiva, L. B. \& Morales, A. R. - Polímeros, 16, p.136 (2006).

14. García-López, D.; Gobernado-Miltre, I.; Merino, J. C. \& Pastor, J. M. - Polym. Bull., 59, p.667 (2007). http://dx.doi. org/10.1007/s00289-007-0810-9

15. Eteläaho, P.; Nevalainen, K.; Suihkonen, R.; Vuorinen, J.; Hanhi, K. \& Järvelä, P. - Polym. Eng. Sci., 49, p.1438 (2009). http://dx.doi.org/10.1002/pen.21270

16. Rodrigues, A. W. B. - "Organofilização de argilas bentoníticas $e$ aplicação no desenvolvimento de nanocompósitos com matriz de polipropileno", Tese de Doutorado, Universidade Federal de Campina Grande, Brasil (2009).

17. Carastan, D. J. - "Obtenção e caracterização reológica de nanocompósitos de polímeros estirênicos”, Tese de Doutorado, Universidade de São Paulo, Brasil (2007).

18. Mohan, T. P. \& Kanny, K. - Engineering, 2, p.802 (2010). http://dx.doi.org/10.4236/eng.2010.210103

19. Xie, S.; Harkin-Jones, E.; Shen, Y.; Hornsby, P.; McAfee, M.; McNally, T.; Patel, R.; Benkreira, H.; Coates, P. - Mater. Lett., 64, p.185 (2010). http://dx.doi.org/10.1016/j. matlet.2009.10.042

Enviado: 18/10/12 Reenviado: 12/12/12 Aceito: 07/01/13 